\title{
Effect of Feeding Rice Based Distillers Dried Grains Solubles with and without Enzymes on Gut health of Broiler Chickens
}

\author{
Om Prakash Dinani*, Pramod Kumar Tyagi, J.S. Tyagi and J.J. Rokade \\ Avian Nutrition and Feed Technology Division, ICAR-Central Avian Research Institute, U.P, INDIA \\ *Corresponding author: OP Dinani; E-mail:dr_dinani@rediffmail.com
}

Received: 02 May, 2020

Revised: 10 Nov., 2020

Accepted: 16 Nov., 2020

\begin{abstract}
A biological experiment was conducted to evaluate the effect of feeding rice based distillers dried grain with solubles (rDDGS) without or with different enzymes on gut health in broiler chickens for 42 days. The experiment was conducted as per $3 \times 4$ factorial completely randomized design. A total of 384 broiler chicks were divided into twelve different treatments with 4 replicates for each treatment and each replicate consisted of 8 chicks. Two levels of rDDGS were taken (12.5 and 15\%). Protease, xylanase and multienzymes supplementation under different treatments were done. The jejunum histomorphometry in terms of villus height $(\mathrm{VH})$ and their ratio with villus depth (VD) were found significantly ( $\mathrm{P}<0.01)$ lower at $15 \%$ rDDGS level compared to control and $12.5 \%$ levels. The xylanase and protease enzyme supplementation significantly $(\mathrm{P}<0.01)$ increased the VH and their ratio with VD as compared to control and multienzymes supplemented group. The interaction effect rDDGS and enzyme supplementation was found on VH and VD ratio, where higher ratio was observed at $12.5 \%$ rDDGS level with protease enzyme and lower ratio was observed at $15 \%$ rDDGS level without enzyme supplementation. The microbiology of the gut in terms of total viable count (TVC) of crop and jejunum significantly $(\mathrm{P}<0.01)$ decreased upon increasing rDDGS levels compared to control, whereas reverse effect was observed on Lactobacillus. Thus, it may be concluded that enzymes supplementation improved histomorphometry and microbiology of the gut in broiler chickens at inclusion level of $12.5 \%$ rDDGS.
\end{abstract}

\section{HIGHLIGHTS}

0 The rDDGS inclusion improved the beneficial microbes in the gut.

(0 Enzymes supplementation improved histomorphometry and microbiology of the gut in broiler chickens at inclusion level of $12.5 \%$ rDDGS.

Keywords: Enzyme, Gut health, Histomorphometry, Rice based distillers dried grain with solubles.

Poultry production in India has taken a quantum leap in the last four decades. Feed is the major component in the production of poultry, as it constitutes 65 to $75 \%$ of total production cost. Soybean meal is the major proteinic ingredient used in poultry diet. The estimated requirement for soybean meal will be 11.9 million tons (MT) in 2025. However, net deficiency of soybean meal in the country is about 2.5 MT annually (Mandal, 2017). Due to scarcity of soybean at reasonable price, there is need to utilize locally available alternate protein ingredients. However, only a narrow range of feed ingredients are used due to lack of reliable data on their nutritive quality, feeding value and safe or effective level of inclusion.
Rice tops the list of total cereal production in the country. About $92 \%$ of total rice production is used for human food and about $8 \%$ is used for livestock and poultry feed in form of rice bran, deoiled rice bran, rice polish and broken rice. India is the second largest producers of rice in the world after China, producing approximately 109.7 MT rice in 2016-17 (Agriculture Statistics, 2018). Now days, certain newer rice by products are available in appreciable

How to cite this article: Dinani, O.P., Tyagi, P.K., Tyagi, J.S. and Rokade, J.J. (2020). Effect of feeding rice based distillers dried grains solubles with and without enzymes on gut health of broiler chickens. J. Anim. Res., 10(6): 1085-1094.

Source of Support: None; Conflict of Interest: None 
quantities and cheaper rate that can be utilized as protein sources from rice processing industries such as rice based distillers dried grain with solubles (DDGS). The DDGS is co-product of the ethanol industry produced during dry milling process. Its availability is increasing due to higher demand for ethanol as biofuel. Rice as subtract for DDGS is increasing due to relative lower price, increased production and easy availability. Most of the researches were limited to corn, wheat, sorghum, barley DDGS. Scanty researches were done in effect of feeding rice DDGS on gut health in poultry (Gupta, 2016; Rao et al., 2016; Ranjan et al. 2017).In the earlier experiment by the author it was found that $12.5 \% \mathrm{rDDGS}$ can be incorporated for economic broiler production (Dinani et al., 2019). So, in this experiment, 12.5 and $15 \%$ rDDGS without or with different enzymes has been tried to study their effect on gut health.

On the other hand, enzyme supplementations in poultry diets are nutritionally, economically and environmentally justified. It leads to increased feeding value of the dietary raw materials, reduction in the variation of nutrient quality of the diet, increased nutrient digestibility and reduction in water content of the excreta, reduced viscosity of intestinal contents and accelerated rate of passage of digest a through the gastrointestinal tract. Different factors such as diet, age as well as health status of the birds influence the establishment of a particular bacterial colony in the gut (Barnes, 1979). However, limited information is available on the appropriate enzyme or the combination of enzymes that are specific for broiler chicken ration based on cornsoya diet and soybean meal partially replaced with rice DDGS.

\section{MATERIALS AND METHODS}

\section{Experimental diets}

The research work was carried out at the Division of Avian Nutrition and Feed Technology, ICAR-Central Avian Research Institute (CARI), Izatnagar, India. Broilers of CARIBRO-VISHAL (white variety) were used in the study for the period of 42 days. The study was carried out as per the guidelines and approval of institute animal ethical committee (IAEC) and committee for the purpose of control and supervision of experiments on animals (CPCSEA). The IAEC/CPCSEA number is 452/01/ab/ CPCSEA. Corn-soya meal based basal diets to meet ICAR
(2013) standard for broiler chickens were formulated as prestarter (Table 1), starter (Table 2) and finisher (Table 3 ). The diets along with all the used ingredients including rice based distiller's dried grains with solubles (rDDGS) were analysed by standard procedure. Whole mash type of feed was used for this study. Energy, protein, major minerals and limiting amino acids will be kept constant. Isonitrogenous and isocalorific diets were used for experiment. The three commercial enzyme preparations protease $(\mathrm{P})$, xylanase $(\mathrm{X})$ and multienzymes $(\mathrm{M})$ were analyzed for different enzyme activities as per standard methods and used as per manufacturer's instruction. The birds were housed in specially designed battery brooder cages with watering and feeding facilities and were reared under standard management conditions.

\section{Experimental design}

The experiment was conducted as per $3 \times 4$ factorial completely randomized design (CRD). A total of 384 broiler chicks (CARIBRO vishal) of same hatch with uniform weight were used in the experiment. The birds were randomly divided into 48 replicates of eight birds each. There were twelve different treatments with 4 replicates for each treatment. So, each treatment was allocated 32 birds. The allocation of birds in each treatment was based on the similar initial body weight. Two levels of rDDGS were taken, the best inclusion level from earlier experiments as first level (12.5\%) and then adding over and above the best level of $2.5 \%$ rDDGS to this level with enzymes. Experimental layout for feeding different level of rDDGS with or without enzymes is presented in Table 4

\section{Intestinal histomorphology}

Samples from jejunum were taken from four birds per treatment at the end of experiment (42 days). Histological slides were prepared by standard procedure. There was one sample for jejunum, two cross-sections per sample and 4 measurements per cross- section (for a total of 32 measurements for each of the jejunum segment per treatment). Four intact villi and crypts of Lieberkuhn were used to give the mean for each chicken. Finally, the mean from four chickens were expressed as the mean villus for one treatment group. Each jejunum segment fixed in $10 \%$ neutral buffered formalin embedded in paraffin 
Table 1: Ingredients and nutrient composition (\%) of pre starter diets with or without enzymes for different level of rDDGS

\begin{tabular}{|c|c|c|c|c|c|c|c|c|c|c|c|c|}
\hline Ingredients & D1 & D2 & D3 & D4 & D5 & D6 & D7 & D8 & D9 & D10 & D11 & D12 \\
\hline Maize & 54.42 & 54.42 & 54.42 & 54.42 & 55.94 & 55.94 & 55.94 & 55.94 & 56.40 & 56.40 & 56.40 & 56.40 \\
\hline SBM & 38.40 & 38.40 & 38.40 & 38.40 & 25.50 & 25.50 & 25.50 & 25.50 & 22.90 & 22.90 & 22.90 & 22.90 \\
\hline DDGS & 0.00 & 0.00 & 0.00 & 0.00 & 12.50 & 12.50 & 12.50 & 12.50 & 15.00 & 15.00 & 15.00 & 15.00 \\
\hline Oil & 3.00 & 3.00 & 3.00 & 3.00 & 1.80 & 1.80 & 1.80 & 1.80 & 1.52 & 1.52 & 1.52 & 1.52 \\
\hline LSP & 1.40 & 1.40 & 1.40 & 1.40 & 1.30 & 1.30 & 1.30 & 1.30 & 1.20 & 1.20 & 1.20 & 1.20 \\
\hline DCP & 1.82 & 1.82 & 1.82 & 1.82 & 1.83 & 1.83 & 1.83 & 1.83 & 1.83 & 1.83 & 1.83 & 1.83 \\
\hline Lysine & 0.00 & 0.00 & 0.00 & 0.00 & 0.23 & 0.23 & 0.23 & 0.23 & 0.27 & 0.27 & 0.27 & 0.27 \\
\hline Methionine & 0.20 & 0.20 & 0.20 & 0.20 & 0.13 & 0.13 & 0.13 & 0.13 & 0.11 & 0.11 & 0.11 & 0.11 \\
\hline Constant* & 0.765 & 0.765 & 0.765 & 0.765 & 0.765 & 0.765 & 0.765 & 0.765 & 0.765 & 0.765 & 0.765 & 0.765 \\
\hline Enzyme & - & + & + & + & - & + & + & + & - & + & + & + \\
\hline Total & 100.01 & 100.01 & 100.01 & 100.01 & 100.00 & 100.00 & 100.00 & 100.00 & 100.00 & 100.00 & 100.00 & 100.00 \\
\hline \multicolumn{13}{|c|}{ Nutrient composition } \\
\hline$\overline{\mathrm{CP}}$ & 21.99 & 21.99 & 21.99 & 21.99 & 22.01 & 22.01 & 22.01 & 22.01 & 22.02 & 22.02 & 22.02 & 22.02 \\
\hline Lysine & 1.19 & 1.19 & 1.19 & 1.19 & 1.23 & 1.23 & 1.23 & 1.23 & 1.23 & 1.23 & 1.23 & 1.23 \\
\hline Methionine & 0.52 & 0.52 & 0.52 & 0.52 & 0.53 & 0.53 & 0.53 & 0.53 & 0.52 & 0.52 & 0.52 & 0.52 \\
\hline Threonine & 0.83 & 0.83 & 0.83 & 0.83 & 0.82 & 0.82 & 0.82 & 0.82 & 0.82 & 0.82 & 0.82 & 0.82 \\
\hline $\mathrm{Ca}$ & 1.03 & 1.03 & 1.03 & 1.03 & 1.05 & 1.05 & 1.05 & 1.05 & 1.03 & 1.03 & 1.03 & 1.03 \\
\hline $\mathrm{P}$ & 0.45 & 0.45 & 0.45 & 0.45 & 0.45 & 0.45 & 0.45 & 0.45 & 0.45 & 0.45 & 0.45 & 0.45 \\
\hline $\operatorname{ME}(\mathrm{kcal} / \mathrm{kg})^{* *}$ & 2998 & 2998 & 2998 & 2998 & 2998 & 2998 & 2998 & 2998 & 2999 & 2999 & 2999 & 2999 \\
\hline $\operatorname{Cost}(₹ / \mathrm{kg})$ & 28.52 & 29.03 & 29.13 & 28.93 & 26.36 & 26.86 & 26.96 & 26.76 & 25.86 & 26.37 & 26.47 & 26.27 \\
\hline
\end{tabular}

In prestarter diet *Constant 0.765 includes salt $0.4 \%$, trace mineral premix $0.1 \%$, vitamin premix $0.15 \%$, vit. B complex $0.015 \%$, choline chloride $0.05 \%$ and Toxin binder $0.05 \%$. Trace mineral premix supplied mg / kg diet: Mn, 55; I, 1; Fe, 75; Zn, 60; Cu, 10; $\mathrm{Se}, 0.15$ and $\mathrm{Cr}$, 0.2. The vitamin premix supplied per kg diet: Vit. A, 5000 IU; Vit. D 3 , 2400 IU; Vit. E, 15 and Vit. K, $1 \mathrm{mg}$. Vitamin B complex supplied per kg diet: Vit. $B_{1}, 5 \mathrm{mg}$; Vit. $\mathrm{B}_{2}, 6 \mathrm{mg}$; Vit. $\mathrm{B}_{6} 5 \mathrm{mg}$; Vit. $\mathrm{B}_{12}, 15 \mathrm{mcg}$; nicotinic acid, $35 \mathrm{mg}$; pantothenic acid, $12 \mathrm{mg}$; biotin $0.15 \mathrm{mg}$ and folic acid $0.5 \mathrm{mg}$. Choline chloride supplied per kg diet: choline, $1300 \mathrm{mg}$. (As per ICAR, 2013)**calculated value.

Table 2: Ingredients and nutrient composition (\%) of starter diets with or without enzymes for different level of rDDGS

\begin{tabular}{|c|c|c|c|c|c|c|c|c|c|c|c|c|}
\hline Ingredients & D1 & D2 & D3 & D4 & D5 & D6 & D7 & D8 & D9 & D10 & D11 & D12 \\
\hline Maize & 55.63 & 55.63 & 55.63 & 55.63 & 57.66 & 57.66 & 57.66 & 57.66 & 58.10 & 58.10 & 58.10 & 58.10 \\
\hline SBM & 37.10 & 37.10 & 37.10 & 37.10 & 24.10 & 24.10 & 24.10 & 24.10 & 21.40 & 21.40 & 21.40 & 21.40 \\
\hline DDGS & 0.00 & 0.00 & 0.00 & 0.00 & 12.50 & 12.50 & 12.50 & 12.50 & 15.00 & 15.00 & 15.00 & 15.00 \\
\hline Oil & 3.50 & 3.50 & 3.50 & 3.50 & 2.15 & 2.15 & 2.15 & 2.15 & 1.90 & 1.90 & 1.90 & 1.90 \\
\hline LSP & 1.35 & 1.35 & 1.35 & 1.35 & 1.20 & 1.20 & 1.20 & 1.20 & 1.17 & 1.17 & 1.17 & 1.17 \\
\hline DCP & 1.55 & 1.55 & 1.55 & 1.55 & 1.58 & 1.58 & 1.58 & 1.58 & 1.58 & 1.58 & 1.58 & 1.58 \\
\hline Lysine & 0.00 & 0.00 & 0.00 & 0.00 & 0.00 & 0.00 & 0.00 & 0.00 & 0.05 & 0.05 & 0.05 & 0.05 \\
\hline Methionine & 0.10 & 0.10 & 0.10 & 0.10 & 0.04 & 0.04 & 0.04 & 0.04 & 0.03 & 0.03 & 0.03 & 0.03 \\
\hline Constant* & 0.765 & 0.765 & 0.765 & 0.765 & 0.765 & 0.765 & 0.765 & 0.765 & 0.765 & 0.765 & 0.765 & 0.765 \\
\hline Enzyme & - & + & + & + & - & + & + & + & - & + & + & + \\
\hline Total & 100.00 & 100.00 & 100.00 & 100.00 & 100.00 & 100.00 & 100.00 & 100.00 & 100.00 & 100.00 & 100.00 & 100.00 \\
\hline \multicolumn{13}{|c|}{ Nutrient composition } \\
\hline $\mathrm{CP}$ & 21.52 & 21.52 & 21.52 & 21.52 & 21.54 & 21.54 & 21.54 & 21.54 & 21.50 & 21.50 & 21.50 & 21.50 \\
\hline Lysine & 1.38 & 1.38 & 1.38 & 1.38 & 1.11 & 1.11 & 1.11 & 1.11 & 1.10 & 1.10 & 1.10 & 1.10 \\
\hline Methionine & 0.48 & 0.48 & 0.48 & 0.48 & 0.48 & 0.48 & 0.48 & 0.48 & 0.49 & 0.49 & 0.49 & 0.49 \\
\hline Threonine & 0.78 & 0.78 & 0.79 & 0.79 & 0.81 & 0.80 & 0.80 & 0.81 & 0.81 & 0.81 & 0.81 & 0.81 \\
\hline $\mathrm{Ca}$ & 0.95 & 0.95 & 0.95 & 0.95 & 0.96 & 0.96 & 0.96 & 0.96 & 0.96 & 0.96 & 0.96 & 0.96 \\
\hline $\mathrm{P}$ & 0.41 & 0.41 & 0.41 & 0.41 & 0.41 & 0.41 & 0.41 & 0.41 & 0.41 & 0.41 & 0.41 & 0.41 \\
\hline $\mathrm{ME}(\mathrm{kcal} / \mathrm{kg})^{* *}$ & 3050 & 3050 & 3050 & 3050 & 3052 & 3052 & 3052 & 3052 & 3053 & 3053 & 3053 & 3053 \\
\hline Cost (₹/kg) & 28.03 & 28.53 & 28.63 & 28.43 & 25.34 & 25.85 & 25.95 & 25.75 & 24.92 & 25.42 & 25.52 & 25.32 \\
\hline
\end{tabular}

In starter diet $*$ Constant 0.765 includes salt $0.4 \%$, trace mineral premix $0.1 \%$, vitamin premix $0.15 \%$, vit. B complex $0.015 \%$, choline chloride $0.05 \%$ and Toxin binder $0.05 \%$. Trace mineral premix supplied $\mathrm{mg} / \mathrm{kg}$ diet: $\mathrm{Mn}, 55 ; \mathrm{I}, 1 ; \mathrm{Fe}, 60 ; \mathrm{Zn}, 60 ; \mathrm{Cu}, 10 ; \mathrm{Se}, 0.15 \mathrm{and} \mathrm{Cr}$, 0.2. The vitamin premix supplied per kg diet: Vit. A, $5000 \mathrm{IU}$; Vit. D, $2400 \mathrm{IU}$; Vit. E, 15 and Vit. K, $1 \mathrm{mg}$. Vitamin B complex supplied per kg diet: Vit. $\mathrm{B}_{1}, 4 \mathrm{mg}$; Vit. $\mathrm{B}_{2}, 6 \mathrm{mg}$; Vit. $\mathrm{B}_{6} 5 \mathrm{mg}$; Vit. $\mathrm{B}_{12}, 15 \mathrm{mcg}$; nicotinic acid, $35 \mathrm{mg}$; pantothenic acid, $10 \mathrm{mg}$; biotin $0.15 \mathrm{mg}$ and folic acid $0.5 \mathrm{mg}$. Choline chloride supplied per kg diet: choline, $1200 \mathrm{mg}$. (As per ICAR, 2013) **calculated value. 
Dinani et al.

Table 3: Ingredients and nutrient composition (\%) of finisher diets for with or without enzymes different level of rDDGS

\begin{tabular}{|c|c|c|c|c|c|c|c|c|c|c|c|c|}
\hline Ingredients & D1 & D2 & D3 & D4 & D5 & D6 & D7 & D8 & D9 & D10 & D11 & D12 \\
\hline Maize & 62.00 & 62.00 & 62.00 & 62.00 & 64.18 & 64.18 & 64.18 & 64.18 & 64.38 & 64.38 & 64.38 & 64.38 \\
\hline SBM & 31.30 & 31.30 & 31.30 & 31.30 & 18.20 & 18.20 & 18.20 & 18.20 & 15.70 & 15.70 & 15.70 & 15.70 \\
\hline DDGS & 0.00 & 0.00 & 0.00 & 0.00 & 12.50 & 12.50 & 12.50 & 12.50 & 15.00 & 15.00 & 15.00 & 15.00 \\
\hline Oil & 3.22 & 3.22 & 3.22 & 3.22 & 1.80 & 1.80 & 1.80 & 1.80 & 1.60 & 1.60 & 1.60 & 1.60 \\
\hline LSP & 1.20 & 1.20 & 1.20 & 1.20 & 1.00 & 1.00 & 1.00 & 1.00 & 0.96 & 0.96 & 0.96 & 0.96 \\
\hline $\mathrm{DCP}$ & 1.45 & 1.45 & 1.45 & 1.45 & 1.50 & 1.50 & 1.50 & 1.50 & 1.50 & 1.50 & 1.50 & 1.50 \\
\hline Lysine & 0.00 & 0.00 & 0.00 & 0.00 & 0.05 & 0.05 & 0.05 & 0.05 & 0.10 & 0.10 & 0.10 & 0.10 \\
\hline Methionine & 0.06 & 0.06 & 0.06 & 0.06 & 0.00 & 0.00 & 0.00 & 0.00 & 0.00 & 0.00 & 0.00 & 0.00 \\
\hline Enzyme & - & + & + & + & - & + & + & + & - & + & + & + \\
\hline Constant* & 0.765 & 0.765 & 0.765 & 0.765 & 0.765 & 0.765 & 0.765 & 0.765 & 0.765 & 0.765 & 0.765 & 0.765 \\
\hline Total & 100.00 & 100.00 & 100.00 & 100.00 & 100.00 & 100.00 & 100.00 & 100.00 & 100.00 & 100.00 & 100.00 & 100.00 \\
\hline \multicolumn{13}{|c|}{ Nutrient composition } \\
\hline $\mathrm{CP}$ & 19.51 & 19.51 & 19.51 & 19.51 & 19.50 & 19.50 & 19.50 & 19.50 & 19.53 & 19.53 & 19.53 & 19.53 \\
\hline Lysine & 1.20 & 1.20 & 1.20 & 1.20 & 0.98 & 0.98 & 0.98 & 0.98 & 0.98 & 0.98 & 0.98 & 0.98 \\
\hline Methionine & 0.41 & 0.41 & 0.41 & 0.41 & 0.42 & 0.42 & 0.42 & 0.42 & 0.43 & 0.43 & 0.43 & 0.43 \\
\hline Threonine & 0.68 & 0.68 & 0.68 & 0.68 & 0.69 & 0.69 & 0.69 & 0.69 & 0.70 & 0.70 & 0.70 & 0.70 \\
\hline $\mathrm{Ca}$ & 0.86 & 0.86 & 0.86 & 0.86 & 0.85 & 0.85 & 0.85 & 0.85 & 0.85 & 0.85 & 0.85 & 0.85 \\
\hline $\mathrm{P}$ & 0.38 & 0.38 & 0.38 & 0.38 & 0.38 & 0.38 & 0.38 & 0.38 & 0.38 & 0.38 & 0.38 & 0.38 \\
\hline $\operatorname{ME}(\mathrm{kcal} / \mathrm{kg})^{* *}$ & 3100 & 3100 & 3100 & 3100 & 3099 & 3099 & 3099 & 3099 & 3101 & 3101 & 3101 & 3101 \\
\hline Cost (₹/kg) & 26.71 & 27.22 & 27.32 & 27.12 & 24.08 & 24.58 & 24.68 & 24.48 & 23.78 & 24.28 & 24.38 & 24.18 \\
\hline
\end{tabular}

In finisher diet $*$ Constant 0.77 includes salt $0.4 \%$, trace mineral premix $0.1 \%$, vitamin premix $0.15 \%$, vit. B complex $0.015 \%$, choline chloride $0.05 \%$ and Toxin binder $0.05 \%$. Trace mineral premix supplied mg / kg diet: $\mathrm{Mn}, 50 ; \mathrm{I}, 1 ; \mathrm{Fe}, 50 ; \mathrm{Zn}, 60 ; \mathrm{Cu}, 8 ; \mathrm{Se}, 0.15$ and $\mathrm{Cr}, 0.2$. The vitamin premix supplied per kg diet: Vit. A, 5000 IU; Vit. D, 2400 IU; Vit. E, 15 and Vit. K, 0.8 mg. Vitamin B complex supplied per kg diet: Vit. $\mathrm{B}_{1}, 4 \mathrm{mg}$; Vit. $\mathrm{B}_{2}, 6 \mathrm{mg}$; Vit. $\mathrm{B}_{6} 5 \mathrm{mg}$; Vit. $\mathrm{B}_{12}, 15 \mathrm{mcg}$; nicotinic acid, $30 \mathrm{mg}$; pantothenic acid, $10 \mathrm{mg}$; biotin $0.15 \mathrm{mg}$ and folic acid $0.5 \mathrm{mg}$. Choline chloride supplied per kg diet: choline, $900 \mathrm{mg}$. (As per ICAR, 2013)**calculated value.

and sections of 5-micron thickness of each sample were placed on a glass slide and stained with hematoxyline and eosine for examination (Culling, 1974).

All the light microscopic variables were measured for jejunum of each bird using optical microscope (Motic Inverted microscope, Honkong), at a $10 \times$ magnification, a camera (Motic cam, CMOS, Honkong), and image analysis software (Motic Image 2.0, Honkong). The morphometric indices in each segment evaluation were villus height (height from the tip of the villus to the crypt), crypt depth (crypt depth from the base of the villi to the submucosa), villus width and the villus height to crypt depth ratio.

\section{Microbiological parameters}

At the end of trail (42 days), four birds from each dietary treatment were sacrificed by cervical dislocation. Crop and jejunum scraping were collected in sterile vials for evaluation of total microbial load colonization. Microbial populations were determined by serial dilution $\left(10^{4}\right.$ to $\left.10^{6}\right)$ of crop and jejunum samples in anaerobic diluents before inoculation onto Petri dishes of sterile agar as described by Bryant and Burkey (1953). Total bacterial count and Lactobacilli were grown on nutrient agar and Rogosa SL agar respectively (Deman et al., 1960). One gram crop and jejunum sample were weighed and dissolved in $9 \mathrm{ml}$ sterile normal saline solution (NSS). Diluted $0.5 \mathrm{ml}$ from $10^{4}$ to $10^{6}$ dilutions was taken in sterile Petri dish and $15-20 \mathrm{ml}$ of sterile agar media were poured in each Petri dish. It was mixed gently and allowed to stand until the media solidify. Then plates were incubated in BOD incubator at $37^{\circ} \mathrm{C}$ for $24 \mathrm{~h}$ and Lactobacillus sp. was incubated anaerobically at $37^{\circ} \mathrm{C}$. Plates were counted between 24 and $48 \mathrm{~h}$ after inoculation. Colony forming units (cfu) were defined as distinct colonies measuring at least $1 \mathrm{~mm}$ in diameter and expressed in $\log 10 \mathrm{cfu} / \mathrm{g}$. It was calculated by the formula $\mathrm{cfu} / \mathrm{g}=$ Total No. of colony counted $\times$ Dilution factor $/$ Volume of aliquot taken. 


\section{Statistical analysis}

Data subjected to test of significance as per completely randomized design (CRD) were analyzed for mean, standard errors and analysis of variance by Snedecor and Cochran (1989) using statistical package for social sciences (SPSS) 16.0 version and comparison of means were done using Tukey's test (1949).

\section{RESULTS AND DISCUSSION}

\section{Intestinal histomorphology}

Effects of feeding different levels of rDDGS with or without enzymes on intestinal histomorphometry (in $\mu \mathrm{m}$ ) are presented in Table 5 and Fig. 1. Feeding different levels of rDDGS $(0,12.5$ and $15 \%)$ on crypt depth (CD)

Table 4: Experimental layout for feeding different level of rDDGS with or without enzymes

\begin{tabular}{|c|c|c|c|c|c|}
\hline \multicolumn{3}{|c|}{ Experimental design } & \multicolumn{3}{|c|}{$3 \times 4$ factorial CRD } \\
\hline Treatment & rDDGS (\%) & No. of replicates & Birds/ replication & Total & Enzymes \\
\hline $\mathrm{T} 1$ & 0.0 & 4 & 8 & 32 & - \\
\hline $\mathrm{T} 2$ & 0.0 & 4 & 8 & 32 & Xylanase \\
\hline $\mathrm{T} 3$ & 0.0 & 4 & 8 & 32 & Protease \\
\hline $\mathrm{T} 4$ & 0.0 & 4 & 8 & 32 & Multienzymes \\
\hline T5 & 12.5 & 4 & 8 & 32 & - \\
\hline T6 & 12.5 & 4 & 8 & 32 & Xylanase \\
\hline $\mathrm{T} 7$ & 12.5 & 4 & 8 & 32 & Protease \\
\hline T8 & 12.5 & 4 & 8 & 32 & Multienzymes \\
\hline T9 & 15 & 4 & 8 & 32 & - \\
\hline T10 & 15 & 4 & 8 & 32 & Xylanase \\
\hline T11 & 15 & 4 & 8 & 32 & Protease \\
\hline $\mathrm{T} 12$ & 15 & 4 & 8 & 32 & Multienzymes \\
\hline
\end{tabular}

Table 5: Effect of feeding different level of rDDGS with or without enzymes on intestinal histomorphometry (in $\mu \mathrm{m}$ )

\begin{tabular}{|c|c|c|c|c|c|c|}
\hline Treatment & rDDGS\% & Enzyme & $\begin{array}{l}\text { Villus height } \\
\text { (VH) }\end{array}$ & $\begin{array}{l}\text { Crypt depth } \\
\text { (CD) }\end{array}$ & VH:CD & Villus width \\
\hline $\mathrm{T} 1$ & 0 & - & 1204 & 159 & $7.62^{\mathrm{ab}}$ & 88 \\
\hline $\mathrm{T} 2$ & 0 & $X$ & 1285 & 143 & $9.02^{\mathrm{cd}}$ & 97 \\
\hline $\mathrm{T} 3$ & 0 & $\mathrm{P}$ & 1320 & 159 & $8.41^{b c}$ & 91 \\
\hline $\mathrm{T} 4$ & 0 & M & 1203 & 144 & $8.40^{\mathrm{bc}}$ & 97 \\
\hline $\mathrm{T} 5$ & 12.5 & - & 1212 & 147 & $8.35^{\mathrm{bc}}$ & 99 \\
\hline T6 & 12.5 & $X$ & 1347 & 162 & $8.35^{\mathrm{bc}}$ & 98 \\
\hline $\mathrm{T} 7$ & 12.5 & $\mathrm{P}$ & 1392 & 145 & $9.72^{\mathrm{d}}$ & 98 \\
\hline $\mathrm{T} 8$ & 12.5 & $\mathrm{M}$ & 1238 & 163 & $7.65^{\mathrm{ab}}$ & 87 \\
\hline T9 & 15 & - & 1025 & 158 & $6.52^{\mathrm{a}}$ & 92 \\
\hline $\mathrm{T} 10$ & 15 & $\mathrm{X}$ & 1252 & 149 & $8.37^{\mathrm{bc}}$ & 97 \\
\hline $\mathrm{T} 11$ & 15 & $\mathrm{P}$ & 1144 & 150 & $7.62^{\mathrm{ab}}$ & 99 \\
\hline \multirow[t]{14}{*}{$\mathrm{T} 12$} & 15 & $\mathrm{M}$ & 1151 & 146 & $7.92^{\mathrm{bc}}$ & 100 \\
\hline & & $\begin{array}{l}\text { Pooled SEM } \\
\text { rDDGS }\end{array}$ & 16.57 & 2.00 & 0.14 & 1.31 \\
\hline & & 0 & $1253^{\mathrm{b}}$ & 151 & $8.36^{\mathrm{b}}$ & 93 \\
\hline & & 12.5 & $1297^{\mathrm{b}}$ & 154 & $8.51^{b}$ & 95 \\
\hline & & 15 & $1142^{\mathrm{a}}$ & 151 & $7.61^{\mathrm{a}}$ & 97 \\
\hline & & Enzyme & & & & \\
\hline & & - & $1146^{\mathrm{a}}$ & 154 & $7.50^{\mathrm{a}}$ & 93 \\
\hline & & $\mathrm{X}$ & $1294^{\mathrm{b}}$ & 151 & $8.58^{b}$ & 97 \\
\hline & & $\mathrm{P}$ & $1285^{\mathrm{b}}$ & 151 & $8.58^{b}$ & 96 \\
\hline & & M & $1197^{\mathrm{a}}$ & 151 & $7.99^{\mathrm{ab}}$ & 94 \\
\hline & & Significance & & & & \\
\hline & & rDDGS & $\mathrm{P}<0.01$ & NS & $\mathrm{P}<0.01$ & NS \\
\hline & & Enzyme & $\mathrm{P}<0.01$ & NS & $\mathrm{P}<0.01$ & NS \\
\hline & & Interaction & NS & NS & $\mathrm{P}<0.05$ & NS \\
\hline
\end{tabular}

Values bearing different superscripts within the column differ significantly $*(\mathrm{P}<0.01),{ }^{* *}(\mathrm{P}<0.05)$ and NS: Non-significant $(\mathrm{P}>0.05)$. 
(2)

Dinani et al.

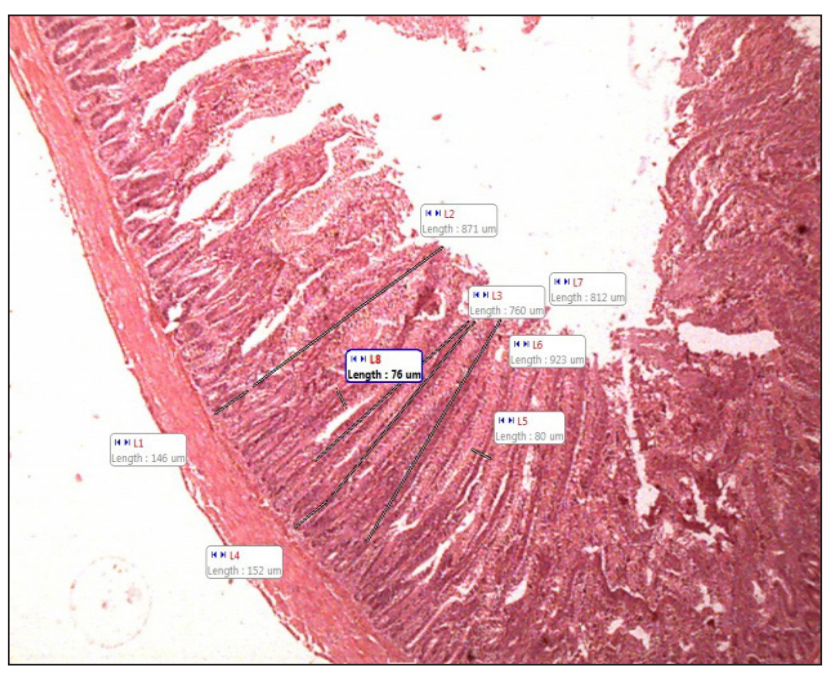

T1

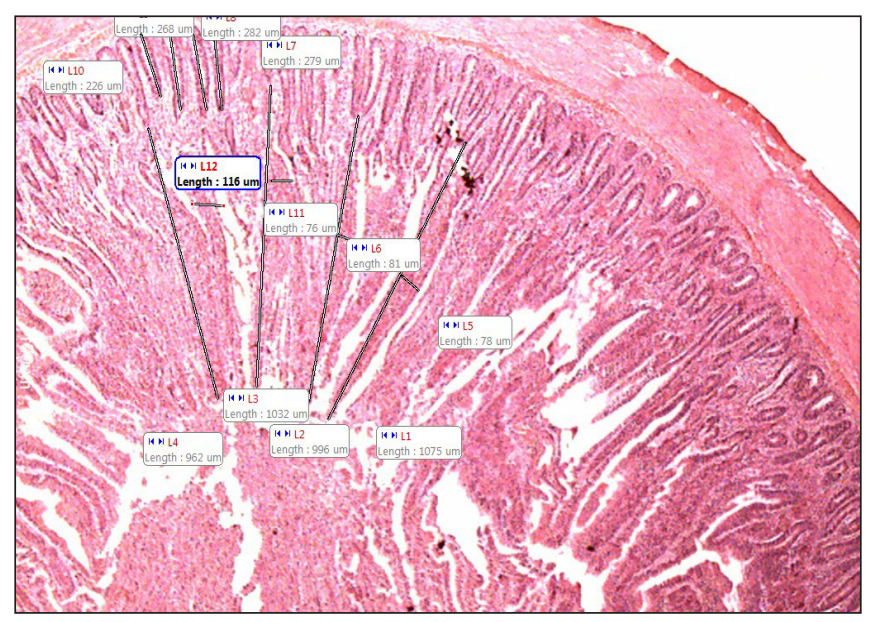

T3

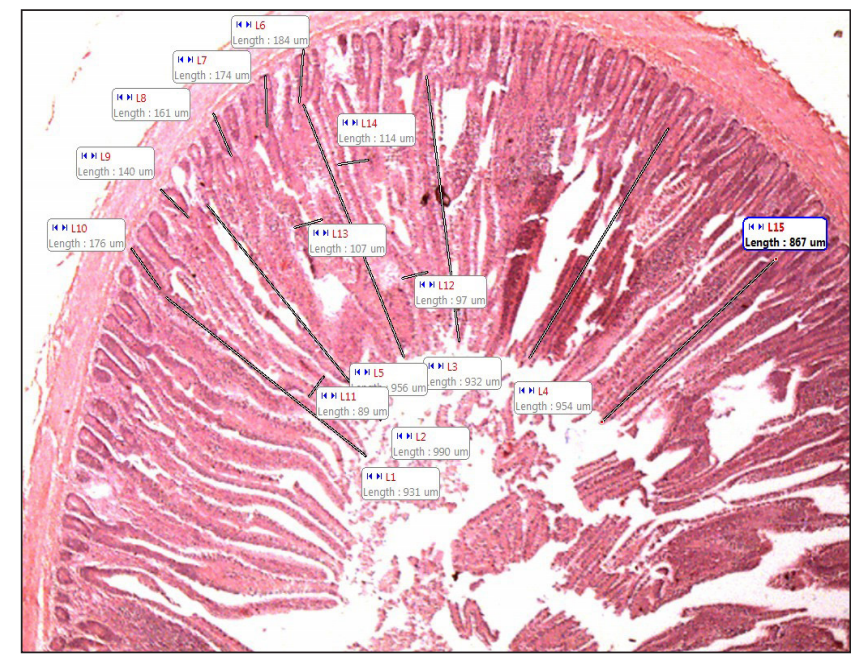

T5

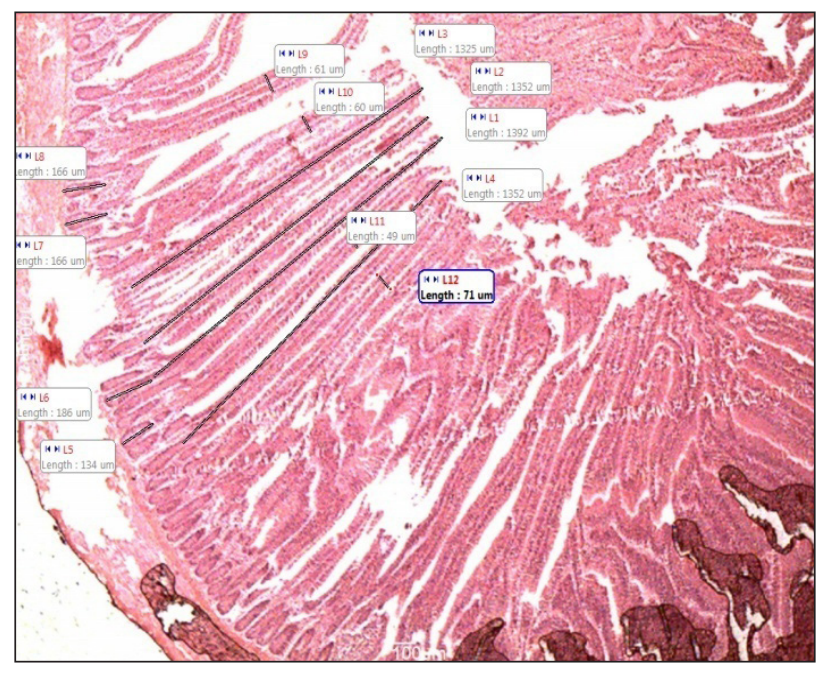

T2

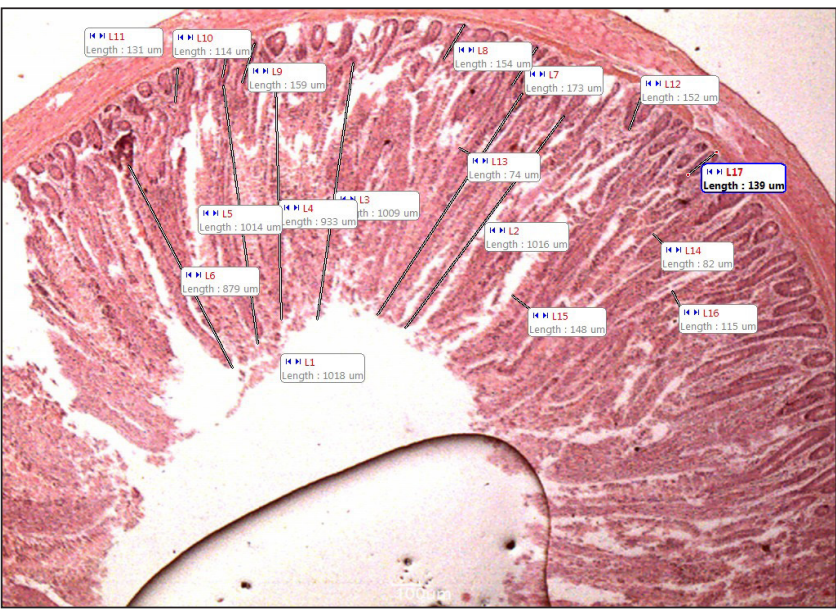

T4

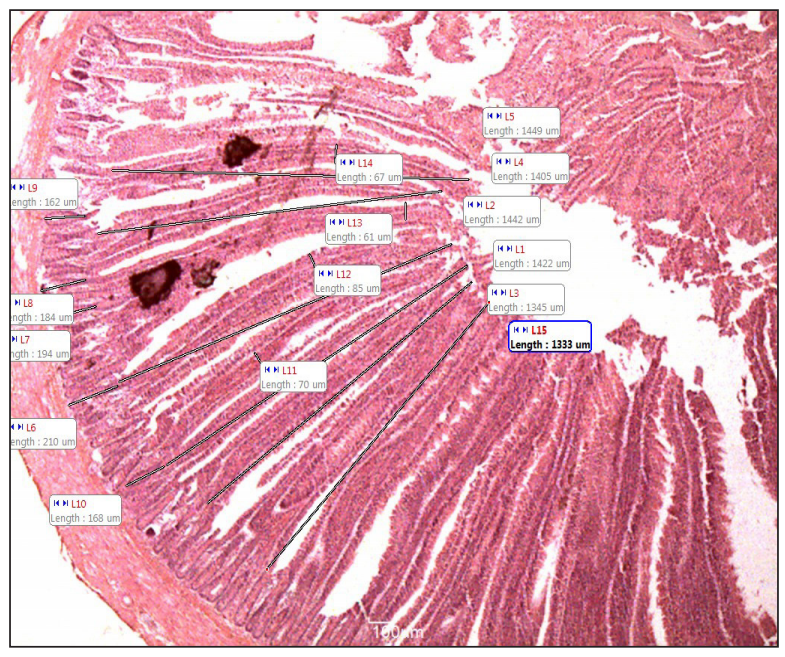

T6 


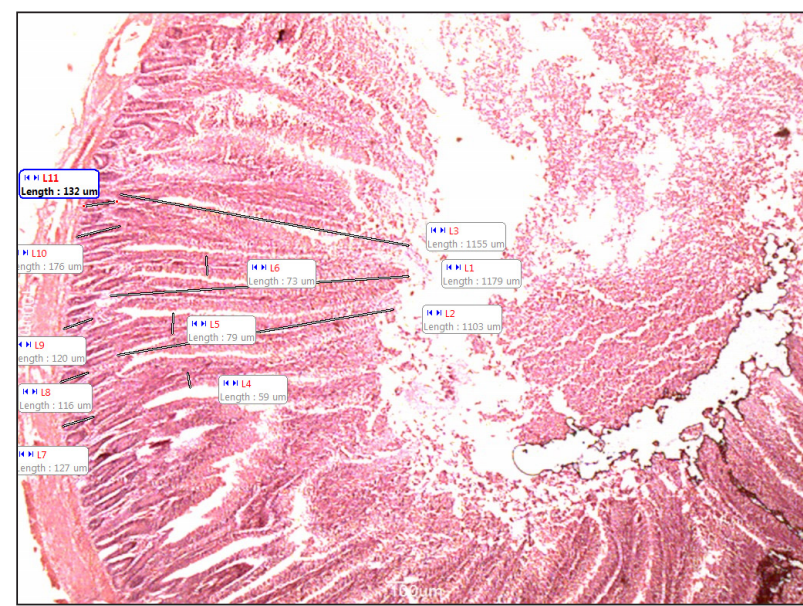

T7

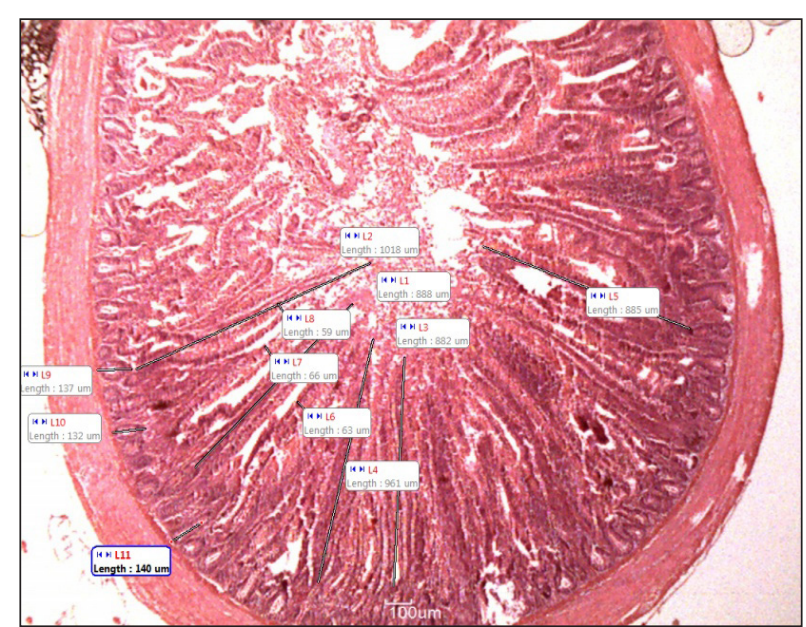

T9

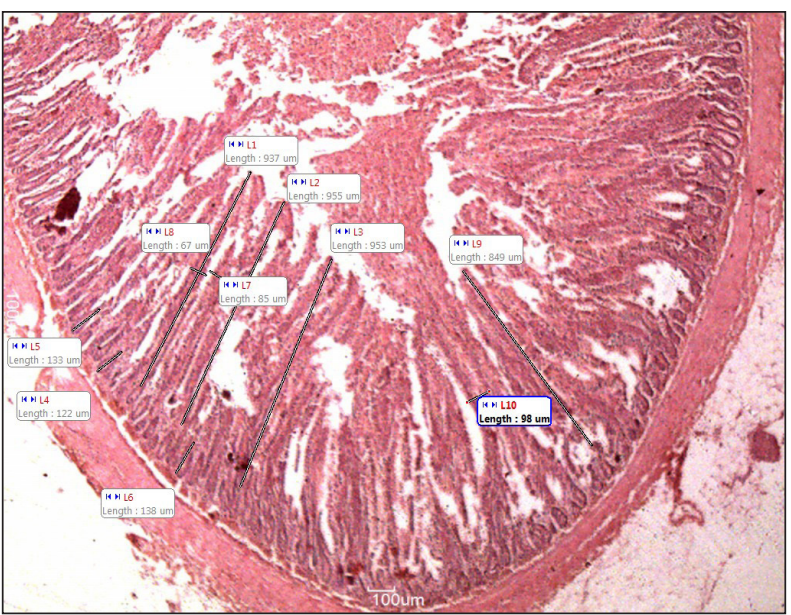

T11

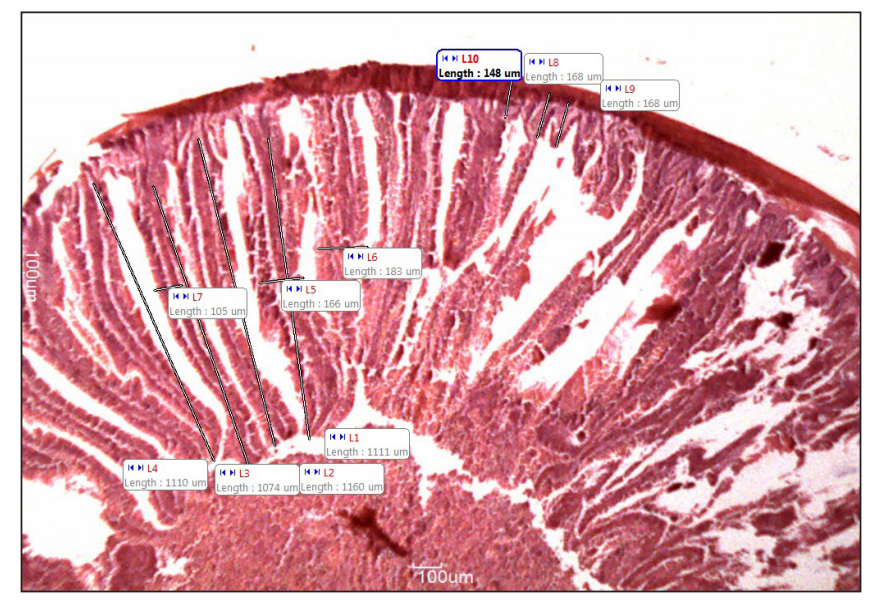

T8

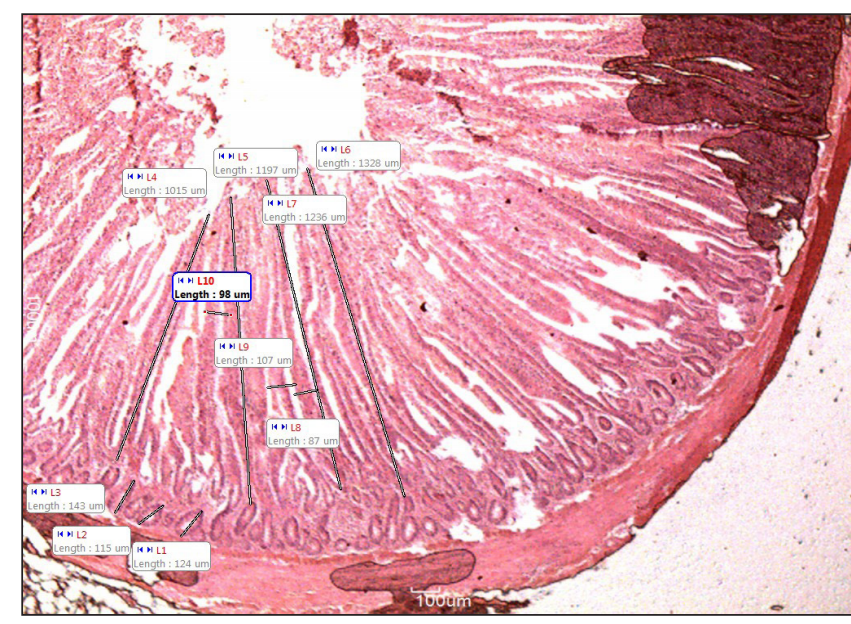

T10

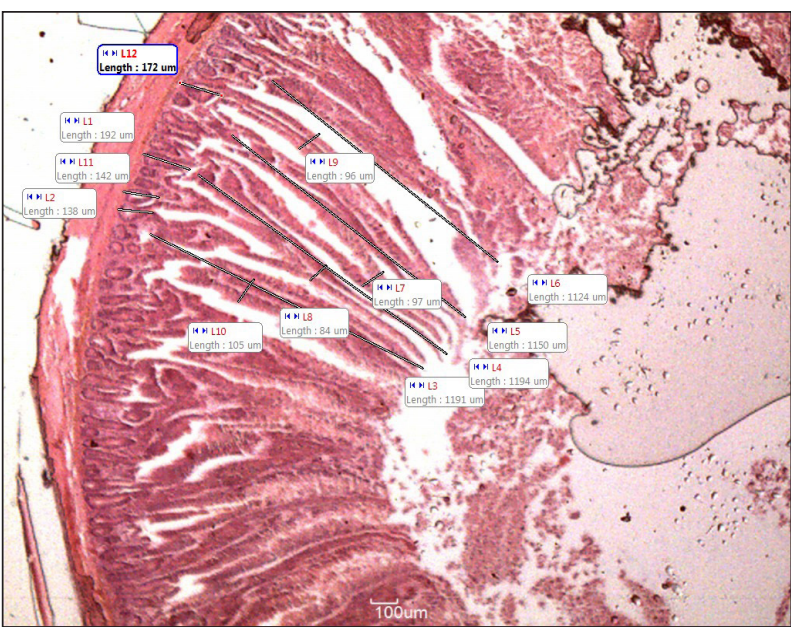

T12

Fig. 1: Effect of feeding different level of rDDGS with or without enzymes on intestinal histomorphometry (in $\mu \mathrm{m}$ ) 
Table 6: Effect of feeding different level of rDDGS with or without enzymes on microbiological parameters $(\log 10 \mathrm{cfu} / \mathrm{g})$

\begin{tabular}{|c|c|c|c|c|c|c|}
\hline \multirow{2}{*}{ Treatment } & \multirow[b]{2}{*}{ rDDGS\% } & \multirow[b]{2}{*}{ Enzyme } & \multicolumn{3}{|c|}{ Crop } & \multirow{2}{*}{$\begin{array}{l}\text { Jejunum } \\
\text { Lactobacillus count }\end{array}$} \\
\hline & & & TVC & Lactobacillus count & TVC & \\
\hline $\mathrm{T} 1$ & 0 & - & 6.80 & 3.37 & 6.74 & 3.52 \\
\hline $\mathrm{T} 2$ & 0 & $\mathrm{X}$ & 6.44 & 3.47 & 6.50 & 3.56 \\
\hline T3 & 0 & $\mathrm{P}$ & 6.63 & 3.21 & 6.54 & 3.75 \\
\hline $\mathrm{T} 4$ & 0 & M & 6.56 & 3.36 & 6.63 & 3.68 \\
\hline T5 & 12.5 & - & 6.20 & 3.77 & 6.33 & 3.82 \\
\hline T6 & 12.5 & $\mathrm{X}$ & 6.23 & 4.01 & 6.31 & 4.25 \\
\hline $\mathrm{T} 7$ & 12.5 & $\mathrm{P}$ & 6.18 & 4.00 & 6.47 & 4.21 \\
\hline $\mathrm{T} 8$ & 12.5 & M & 6.38 & 3.99 & 6.38 & 4.22 \\
\hline T9 & 15 & - & 6.24 & 3.86 & 6.08 & 4.01 \\
\hline $\mathrm{T} 10$ & 15 & $\mathrm{X}$ & 5.96 & 3.98 & 5.96 & 4.04 \\
\hline T11 & 15 & $\mathrm{P}$ & 6.07 & 4.00 & 6.35 & 4.16 \\
\hline \multirow[t]{14}{*}{$\mathrm{T} 12$} & 15 & M & 6.20 & 3.84 & 6.20 & 4.11 \\
\hline & & $\begin{array}{l}\text { Pooled SEM } \\
\text { rDDGS }\end{array}$ & 0.04 & 0.05 & 0.04 & 0.04 \\
\hline & & 0 & $6.60^{c}$ & $3.35^{\mathrm{a}}$ & $6.60^{c}$ & $3.62^{\mathrm{a}}$ \\
\hline & & 12.5 & $6.24^{b}$ & $3.94^{\mathrm{b}}$ & $6.37^{b}$ & $4.12^{b}$ \\
\hline & & 15 & $6.11^{\mathrm{a}}$ & $3.92^{\mathrm{b}}$ & $6.14^{\mathrm{a}}$ & $4.07^{b}$ \\
\hline & & Enzyme & & & & \\
\hline & & - & $6.42^{c}$ & 3.67 & $6.38^{\mathrm{ab}}$ & $3.78^{a}$ \\
\hline & & $X$ & $6.19^{a}$ & 3.82 & $6.25^{\mathrm{a}}$ & $3.95^{\mathrm{b}}$ \\
\hline & & $\mathrm{P}$ & $6.29^{\mathrm{ab}}$ & 3.74 & $6.45^{b}$ & $4.04^{b}$ \\
\hline & & $\mathrm{M}$ & $6.38^{c}$ & 3.73 & $6.40^{\mathrm{b}}$ & $4.00^{\mathrm{b}}$ \\
\hline & & Significance & & & & \\
\hline & & rDDGS & $\mathrm{P}<0.01$ & $\mathrm{P}<0.01$ & $\mathrm{P}<0.01$ & $\mathrm{P}<0.01$ \\
\hline & & Enzyme & $\mathrm{P}<0.01$ & NS & $\mathrm{P}<0.05$ & $\mathrm{P}<0.01$ \\
\hline & & Interaction & NS & NS & NS & NS \\
\hline
\end{tabular}

Values bearing different superscripts within the column differ significantly $*(\mathrm{P}<0.01), * *(\mathrm{P}<0.05)$ and NS: Non-significant $(\mathrm{P}>0.05)$.

and villus width (VW) did not exhibit any significant $(\mathrm{P}>0.05)$ difference between different dietary treatments and control. Ratio of villus height $(\mathrm{VH})$ with crypt depth (CD) and villus height were significantly $(\mathrm{P}<0.01)$ lower in $15 \%$ rDDGS level as compared to 0 and $12.5 \%$ levels.

Effect of feeding rDDGS without or with enzymes (xylanase, protease and multienzymes) on intestinal histomorphometry did not exhibit any significant $(\mathrm{P}>0.05)$ difference on crypt depth and villus width. Xylanase and protease enzyme supplementation significantly $(\mathrm{P}<0.01)$ increased villus height compared to multienzymes and without enzyme groups. Xylanase and protease enzyme supplementation also significantly $(\mathrm{P}<0.01)$ increased $\mathrm{VH}$ : $\mathrm{CD}$ ratio as compared to without enzyme groups, but $\mathrm{VH}$ : $\mathrm{CD}$ ratio did not show any significant $(\mathrm{P}>0.05)$ difference as compared to multienzymes supplementation.

Interaction of rDDGS and enzymes did not show any significant $(\mathrm{P}>0.05)$ difference in villus height, crypt depth and villus width as compared to control and other dietary treatments except on $\mathrm{VH}$ : $\mathrm{CD}$ ratio. Ratio of $\mathrm{VH}$ and CD significantly $(\mathrm{P}<0.05)$ increased in $0 \%$ rDDGS with xylanase and $12.5 \% \mathrm{rDDGS}$ with protease as compared to control and other dietary treatments.

Information on effect of feeding diets containing various levels of rice DDGS on histological changes in broiler are very scanty in literature. However, our results are in agreement with Loar et al. (2010) and Gupta et al. (2015), but disagreement with Ranjan et al. (2017). Loar et al. (2010) reported no adverse effect on intestinal parameters by incorporating DDGS in post hatch 28 days broilers. Gupta (2016) reported no negative effects on intestinal morphology up to $10 \%$ inclusion of rDDGS and protease supplementation was beneficial for villus height, width, crypt depth and VH: CD ratio. Contrary to this, Ranjan et al. (2017) reported that no significant $(\mathrm{P}>0.05)$ difference in villus length and crypt depth in duodenum, jejunum and ileum in duck fed various levels of rice DDGS up to $75 \%$ replacement of soybean meal. Decrease in villus length in 
$15 \%$ rDDGS may be associated with poor digestibility at this inclusion level.

\section{Microbiological parameters}

The results pertaining to influence of different levels of rDDGS with or without enzymes on total viable count (TVC) and Lactobacillus count (log $10 \mathrm{cfu} / \mathrm{g})$ in crop and jejunum are presented in Table 6.

Feeding different levels of rDDGS $(0,12.5$ and $15 \%)$ on crop and jejunum significantly $(\mathrm{P}<0.01)$ decreased TVC at 12.5 and $15 \%$ rDDGS levels, but $15 \%$ rDDGS level significantly $(\mathrm{P}<0.01)$ decreased TVC as compared to 0 and $12.5 \%$ rDDGS levels. Lactobacillus count on crop and jejunum both significantly $(\mathrm{P}<0.01)$ increased at 12.5 and $15 \%$ rDDGS levels, but $15 \%$ rDDGS level significantly $(\mathrm{P}<0.01)$ increased Lactobacillus count as compared $12.5 \%$ rDDGS level.

Enzymes supplementation (xylanase, protease and multienzymes) did not show any significant $(\mathrm{P}>0.05)$ difference in Lactobacillus count in crop. Xylanase supplementation significantly $(\mathrm{P}<0.01)$ decreased TVC as compared to without or with enzymes (protease and multienzymes) in crop. Xylanase supplementation also significantly $(\mathrm{P}<0.01)$ decreased TVC in jejunum as compared to protease and multienzymes supplementation, but it did not show any significant $(\mathrm{P}>0.05)$ difference from without enzyme groups. Lactobacillus count significantly $(\mathrm{P}<0.01)$ increased in jejunum in all enzymes supplemented groups (X, P and $\mathrm{M}$ ) as compared to without enzyme groups. Interaction of rDDGS and enzymes did not show any significant $(\mathrm{P}>0.05)$ difference in microbial population in crop and jejunum.

Our results are coinciding with Hahn (2010), Yang et al. (2010) and Gupta et al. (2015). Hahn (2010) observed that the diets containing corn DDGS had significantly $(\mathrm{P}<0.05)$ higher count of Lactobacillus as compared to control diet. Yang et al. (2010) reported that diets containing corn DDGS had greater lactic acid containing bacteria than diets containing wheat DDGS. Yan et al. (2013) also suggested that addition of NSP degrading enzymes improved gut health in broilers. Gupta et al. (2015) reported rDDGS in layer diet up to the inclusion level of $10 \%$ decreased TVC and improved Lactobacillus count. This could be because of the composition of DDGS in which it is more fibrous and offer more nutrients to Lactobacillus.

\section{CONCLUSION}

Thus, it may be concluded that enzymes supplementation improved histomorphometry and microbiology of the gut by feeding rDDGS in broiler chickens at inclusion level of $12.5 \%$.

\section{ACKNOWLEDGEMENTS}

ICAR-Central avian research institute, Izatnagar, Utter Pradesh-243122 for providing all necessary inputs and facilities.

\section{REFERENCES}

Agricultural Statistics. 2018. Agricultural statistics at a glance. Department of agriculture, cooperation and farmers welfare. Government of India, New Delhi.

Barnes, E.M. 1979. The intestinal microflora of poultry and game birds during life and after storage. J. Appl. Bacteriol., 46: 407-419.

Bryant, M.P. and Burkey L.A. 1953. Cultural methods and some characteristics of some of the more numerous groups of bacteria in the bovine rumen. J. Dairy Sci., 36: 205-217.

Culling, C.F.A. 1974. Handbook of histopathological and histochemical techniques. $3^{\text {rd }}$ edn. Butterworths \& Co., London, pp. 343-49.

Deman, J.D., Rogosa, M. and Sharpe, M.E. 1960. A medium for the cultivation of Lactobacilli. J. Appl. Bacteriol., 23: 130135

Dinani, O.P., Tyagi Pramod, K., Mandal, A.B., Tyagi Praveen, K. and Dutta, N. 2019. Evaluation of feeding value of rice based Distillers Dried Grains with Solubles (DDGS) for broiler chickens. Indian J. Anim. Res., 53(7): 901-906.

Gupta, S.L., Tyagi, Pramod K., Tyagi, Praveen K., Mandal, A.B., Mir, N.A. and Sharma, M. 2015. Intestinal histomorphometry of laying hens fed diets containing rice based dry distiller's grains with solubles. Indian J. Poult. Sci., 50(3): 294-299.

Hahn, Dana L. 2010. The effects of phytase and an enzyme combination in moderate and low nutrient dense diets in laying hens. Thesis and dissertations in animal science, Paper 23.

ICAR. 2013. Nutrient requirements of Animals-Poultry. Indian Council of Agriculture Research, New Delhi, India, 1: 13-16.

Loar, R.E., Moritz, J.S., Donaldson, J.R. and Corzo, A. 2010. Effects of feeding distillers dried grains with soluble to broilers from 0 to 28 days post hatch on broiler performance, feed manufacturing efficiency and selected intestinal characteristics. Poul. Sci., 89: 2242-2250. 
Mandal, A.B. 2017. Challenges of feed industries for sustainable poultry production. IPSACON, Lead paper and souvenir, pp. 93-108.

Ranjan, A., Samanta, Gautam and Samanta, G. 2017. Rice based distiller dried grains and solubles in duck egg production and its quality. Indian J. Poult. Sci., 52(3): 255-258.

Snedecor, G.W. and Cochran, W.G. 1989. Statistical Methods, $7^{\text {th }}$ edn. Oxford and IBH. Iowa State University Press, Iowa, USA.

Tukey, J. 1949. Comparing individual means in the analysis of variance. Biometrics, 5(2): 99-114.
Yan, F., Dibner, J. and Vazquez-Anon, M. 2013. Effect of dried distillers grain with solubles (DDGS) and rye on growth performance and gut health of broilers as affected by enzyme supplementation. Annual Meeting, Knight Novus C. International Inc. St. Charles.

Yang, Y.E., Kiarie, Slominski, B.A., Brule-Babel, A. and Nyachoti, C.M. 2010. Amino acid and fiber digestibility, intestinal bacterial profile, and enzyme activity ingrowing pigs fed dried distillers grains with solubles based diets. J. Anim. Sci., 88: 3304- 3312. 